\title{
Application of Magnetic Ribbon Coated with Poly(3,4-ethylenedioxythio- phene) /Poly(4-styrenesulfonate) to a Wireless Ammonia Sensor
}

\author{
O. Ishii, O. Mori*, K. Takahashi, N. Kutsuzawa, and H. Okuzaki** \\ Department of Electrical Engineering, Yamagata Univ., Johnan 4-3-15, Yonezawa-shi, Yamagata, 992-8510, Japan \\ *Graduate School of Electronic Engineering, Tohoku Gakuin Univ., Chuo 1-13-1, Tagajyo-shi, Miyagi, 985-8537, Japan \\ ** Graduate School of Medicine and Engineering, Univ. Yamanashi, Takeda 4-4-3, Kofu-shi, Yamanashi, 400-8510, Japan
}

This paper describes a wireless ammonia sensor that employs the resonance frequency change in a curved magnetic ribbon coated with poly(3,4-ethylenedioxythiophene) doped with poly(4-styrenesulfonate) (PEDOT/PSS). As PEDOT/PSS expands due to the sorption of ammonia molecules, the curvature of the ribbon is decreased with increasing ammonia concentration, $P_{\mathrm{am}}$. The decrease in curvature reduces the mechanical resonance frequency from $105 \mathrm{kHz}$ at $P_{\mathrm{am}}=0 \%$ to $89 \mathrm{kHz}$ at $P_{\mathrm{a}}=10 \%$. We also confirmed that the resonance frequency is proportional to the curvature and that the proportional constant is $0.33 \mathrm{kHz} / \mathrm{m}^{-1}$. Since the mechanical vibration of the ribbon is excited by applying AC and DC fields and is detected by monitoring the voltage induced on a pickup coil, the $P_{\text {am }}$ value around the ribbon can be detected wirelessly.

Key words: ammonia, sensor, magnetic ribbon, resonance frequency, PEDOT/PSS

\section{Introduction}

Today, many kinds of foods are sold in airtight and tamper-resistant packages. There is a real need to monitor the ammonia concentration, $P_{\mathrm{am}}$, in packages in order to ensure the quality and safety of these foods. Therefore, demand has increased for a disposable wireless ammonia sensor that is small enough to be installed in the packages. Many kinds of ammonia sensors have been developed; most of them are made of ceramic semiconductors, whose impedance changes with a change in $P_{\mathrm{am}}{ }^{1)}$. These ammonia sensors must be connected to electrical circuitry with bonding wires, and this often leads to problems with fabrication processes because the wires are susceptible to mechanical stress. This why the demand has increased for a wireless ammonia sensor. In contrast, ammonia detecting tubes are widely used because of their high sensitivity. When a sample gas is passed through these tubes, their color changes irreversibly in response to $P_{\mathrm{am}}$, which means that the tube can only be used once. Hence, the tubes must be replaced for each measurement, and this has prevented their widespread use. Therefore, the need for a disposable and conveniently designed wireless ammonia sensor has emerged.

We have developed wireless gas sensors that use the mechanical resonance of a highly magnetoelastic ribbon coated with selective gas sensing layers whose volume changes with the gas concentration ${ }^{2,3)}$. The ribbon vibrates mechanically when we apply $\mathrm{AC}$ and $\mathrm{DC}$ magnetic fields. The behavior of the vibration can be detected wirelessly by monitoring the voltage induced in a pickup coil placed at a distance from the ribbon. When the volume of the gas sensing layer changes, the curvature, $1 / R$, of the ribbon also changes. The deformation of the ribbon affects the magnitude and distribution of the magnetic anisotropy as well as the $\Delta E$ effect, which results in great variations in the mechanical resonance frequency, $f r$. Consequently, the gas concentration can be detected wirelessly by monitoring $f r$. Jain et al. reported a similar relationship between the resonance frequency and curvature for magnetoelastic amorphous ribbons ${ }^{4}$; planar (length: $25 \mathrm{~mm}$ ), semi-circular (nominal length: $33 \mathrm{~mm}$ and radius: $10.5 \mathrm{~mm}$ ) and ring-like circular (nominal length: $51.4 \mathrm{~mm}$ and radius: $8.2 \mathrm{~mm}$ ) ribbons yielded almost the same $f r$ values $(\sim 93 \mathrm{kHz})$. The shape-dependent difference in the resonance frequency was interpreted to be due to the bend-related stressing of the magnetostrictive material. Physically bending or curving the material increases its elasticity, acting to shift the resonance frequency upward.

Many kinds of conductive polymers are also being intensively developed for electronic and electromechanical system (MEMS) applications. The volume of conductive polymers generally increases when a dopant is added. Polypyrole (PPy) expands or contracts when it absorbs or desorbs water vapor ${ }^{5)}$ or ammonia molecules ${ }^{6}$. Okuzaki et al. also reported that PEDOT/PSS, which is a commonly used conductive polymer, exhibits a larger change in volume than PPy when the relative humidity is changed $^{7}$. As a result, we speculated that the volume of PEDOT/PSS would also change in response to a change in $P$ am. We investigated the relationship between $f r$ and $1 / R$ within this context for a PEDOT/PSS-coated magnetic ribbon to fabricate a disposable wireless ammonia sensor that could be installed in airtight and tamper-resistant packages. It should be noted that because the ribbon and gas sensing layer are inexpensive, the sensors can be used on a disposable basis. 


\section{Experiments}

Livingston reported that the desired properties for high magnetomechanical sensitivity are a high saturation magnetostrictive constant, $\lambda_{\mathrm{s}}$, spontaneous magnetization, $M_{\mathrm{s}}$, and Young's modulus at constant magnetization (e.g., at saturation), $E_{\mathrm{m}}$, and low (and homogeneous) induced transverse magnetic anisotropy energy, $K_{\mathrm{u}}{ }^{8}$. $\mathrm{Fe}_{82} \mathrm{~B}_{13.5} \mathrm{Si}_{8.5} \mathrm{C}_{2}$ amorphous alloy (Metglas 2605SC) is a typical material that has high $\lambda_{\mathrm{s}}=27 \sim 30 \times 10^{-6}$, $M_{\mathrm{s}}=1.4 \mathrm{~T}$, and $E_{\mathrm{m}}=200 \mathrm{GPa}$ and low $K_{\mathrm{u}}=38 \mathrm{~J} / \mathrm{m}^{3}$ when annealed at $385^{\circ} \mathrm{C}$ for 10 min under a transverse magnetic field ${ }^{9}$. In particular, the $K_{\mathrm{u}}$ value of this material is greatly decreased by applying tensile stress, $\sigma_{x}>0$, in parallel to the ribbon length $(x \text {-axis })^{9)}$; the application of $\sigma_{x}$ on the ribbon contributes anisotropically to the energy density of $-(3 / 2) \lambda_{s} \sigma_{x}$. The tensile stress has the effect of lowering the net anisotropy from $K_{\mathrm{u}}$ to $K_{\mathrm{u}}-(3 / 2) \lambda_{\mathrm{s}} \sigma_{x}$ for $\lambda_{\mathrm{s}} \sigma_{x}>0$, i.e., tensile stress, $\sigma_{x}>0$, on a positive-magnetostriction material or compressive stress, $\sigma_{x}<0$, on a negative-magnetostriction material. The net anisotropy for this material decreases to a minimum value of $\sim 10 \mathrm{~J} / \mathrm{m}^{3}$ as $\sigma_{x}$ increases to the critical stress, $\sigma_{\mathrm{c}}=0.7 \mathrm{MPa}^{8)}$. Low values for the net anisotropy and $\sigma_{\mathrm{c}}$ permit rotation of magnetization and accompanying magnetostrictive strain with low applied fields or stresses, yielding superior magnetomechanical properties. Therefore, we used a $25-\mu \mathrm{m}$ thick Metglas 2605SC ribbon for application to the sensors. We cut the ribbon into a rectangle $(37 \times 6 \mathrm{~mm})$, and then relieved its internal stress by annealing it at $410^{\circ} \mathrm{C}$ for $10 \mathrm{~min}$ in a nitrogen flow. We applied a transverse magnetic field of $7960 \mathrm{~A} / \mathrm{m}$ (100 Oe) with a Helmholtz coil during the annealing to induce uniaxial magnetic anisotropy. A $10-\mu \mathrm{m}$ thick PEDOT/PSS sheet was fabricated using a previously reported method ${ }^{5)}$ and pasted to the ribbon with a commercially available epoxy paste.

Figure 1 outlines the setup to measure the resonance frequency. The coil (diameter: $30 \mathrm{~mm}$, number of turns: 60) was connected to port 1 of a network analyzer (HP8510A). The input power was $1 \mathrm{dBm}(1 \mathrm{~mW})$. A acrylic tube was placed in the coil, and a $\mathrm{PE}^{-}$ DOT/PSS-coated magnetic ribbon was placed in the acrylic tube. A DC bias magnetic field, $H_{\text {dc }}(79.6$ - 1035 $\mathrm{A} / \mathrm{m}, 1-13 \mathrm{Oe}$ ), was applied with a Helmholtz coil. We detected the input power absorption as the reflection coefficient, $S_{11}$, and defined $f r$ as the $S_{11}$ dip point. The $P_{\text {am }}$ value was adjusted by mixing $99.995 \%$ nitrogen gas (dew point $<-61^{\circ} \mathrm{C}$ ) and a mixture of ammonia and nitrogen gases. To determine the optimum $H_{\text {dc }}$ at which a flat magnetic ribbon exhibits minimum $f r$, a wet nitrogen (60 RH\%) flow was also introduced. The total gas flow rate and temperature were about $5 \mathrm{l} / \mathrm{min}$ and $23^{\circ} \mathrm{C}$. The temperature was monitored with a commercially available temperature sensor (Sensirion Ltd, SHT-11).

The $1 / R$ value was defined as the inverse of the radius, $R$, of a circle in contact with the center and both edges of the ribbon. The $R$ value was calculated from the coordi- nates of the pixels of the three points in a digitized side view image of the ribbon in the absence of $\mathrm{AC}$ and $\mathrm{DC}$ magnetic fields.

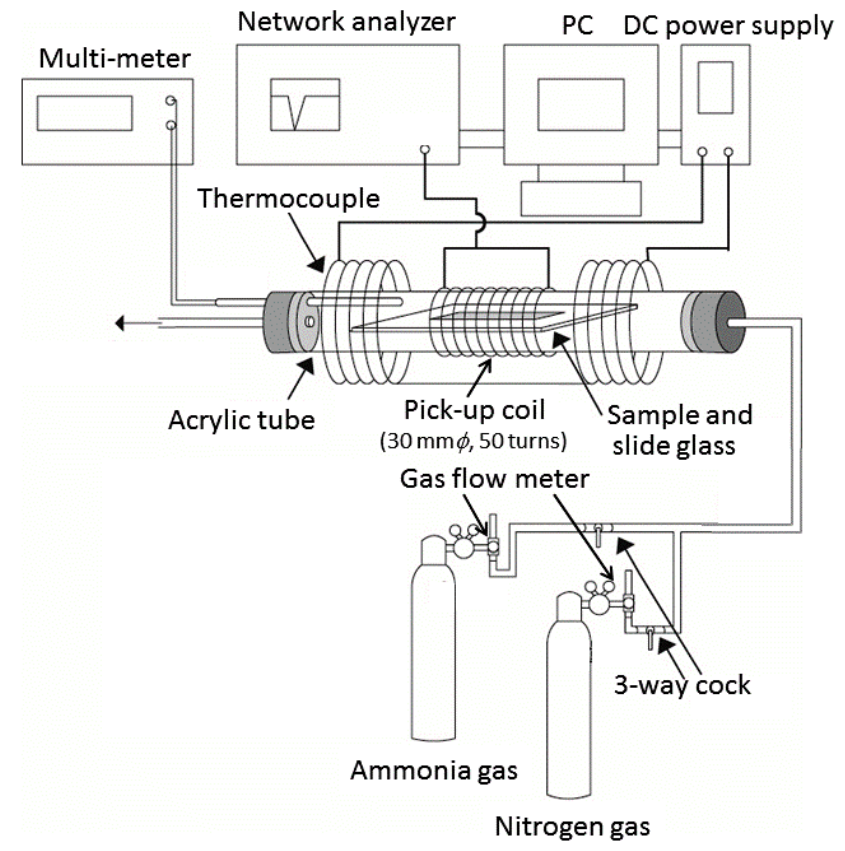

Fig. 1 Resonance frequency measurement setup.

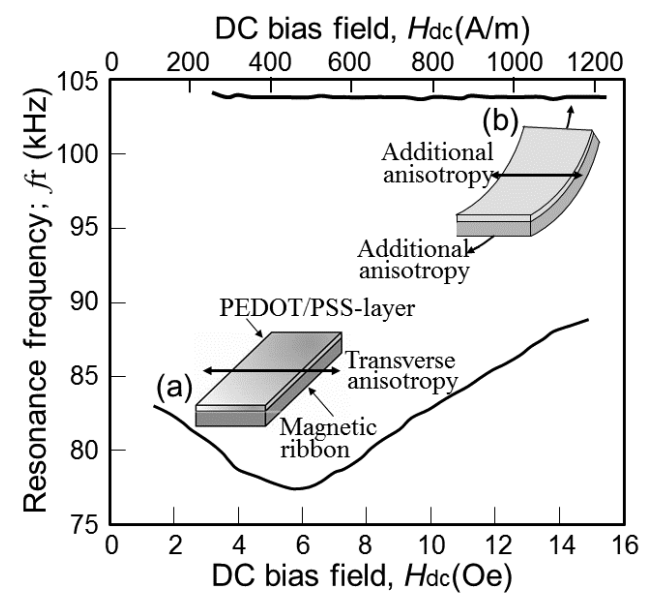

Fig. 2 DC bias field, $H_{\mathrm{dc}}$, dependence of resonance frequency, $f r$, in (a) wet and (b) dry nitrogen atmospheres.

\section{Results and discussion}

Figure 2 plots the dependence of $f r$ on $H_{\text {dc }}$ in dry and wet nitrogen atmospheres. The ribbon was bent and $f r$ was constant $(103.5 \mathrm{kHz})$ in the dry nitrogen atmosphere. In contrast, the ribbon was flat in the wet nitrogen atmosphere and exhibited low $f r$ accompanied by a broad dip at $H_{\mathrm{dc}}=470 \mathrm{~A} / \mathrm{m}(5.9 \mathrm{Oe})$, which indicates a clear $\Delta E$ effect $^{5,6)}$. As a result, the optimum $H_{\text {dc }}$ value to maximize the change in $f r$ was found to be $470 \mathrm{~A} / \mathrm{m}(5.9$ $\mathrm{Oe})$, which is almost equal to the anisotropy field, $H_{\mathrm{a}}=400 \mathrm{~A} / \mathrm{m}$ (5.0 Oe), determined as the "knee" field of the $B-H$ curve of the ribbon. Similar to commercially available magneto-acoustic anti-theft tags ${ }^{10)}, H_{\mathrm{dc}}$ can be supplied by the adjacent placement of magnetically hard 
(large stray field) strips of material, whose amplitude is chosen to minimize $f r$. While it is necessary to apply $H_{\mathrm{dc}}$ along the magnetic hard axis of the ribbon, the AC field can be applied in any orientation. The $H_{\mathrm{dc}}$ value imposed by the adjacent placement of magnetically hard strips was selected to be in the middle of the flat $f r-H_{\mathrm{dc}}$ region to avoid the errors in measurement associated with variable $H_{\mathrm{dc}}$. Our interest relating to sensors was not in the relative changes in the amplitude of the response, but rather in the shifts in $f r$ in response to $P_{\mathrm{am}}$. We eliminated the orientation effects between the sensor and AC field by monitoring the fr of the sensor, rather than the amplitude.

The fundamental resonance frequency, $f$, is generally given by

$$
f=\frac{1}{2 L} \sqrt{\frac{E}{\rho}}
$$

for a thin, planar ribbon of length $L$ that vibrates on its basal plane, where $\rho$ is the density of the ribbon material and $E$ is Young's modulus of elasticity. The change in $E$ for Metglas 2605SC ribbons should be associated with the $\Delta E$ effect, which is largely dependent on internal stress; Livingston reported a simplified model of magnetomechanical coupling as well as the $\Delta E$ effect in amorphous magnetic alloy ribbons with transverse anisotropy $^{8)}$. This model has assumed that $K_{\mathrm{u}}, \lambda_{\mathrm{s}}, M_{\mathrm{s}}$, and $E_{\mathrm{m}}$ are all homogeneous throughout the sample. Because the application of longitudinal tensile stress, $\sigma_{x}>0$, has an effect of lowering the net anisotropy to $K_{\mathrm{u}}-(3 / 2) \lambda_{\mathrm{s}} \sigma_{x}$, the anisotropy field is also decreased from $H_{\mathrm{a}}=2 K_{\mathrm{u}} / M_{\mathrm{s}}$ to $H_{\mathrm{a}_{\sigma}}=\left(2 K_{\mathrm{u}}-3 \lambda_{\mathrm{s}} \sigma_{x}\right) / M_{\mathrm{s}}=H_{\mathrm{a}}-\left(3 \lambda_{\mathrm{s}} \sigma_{x} / M_{\mathrm{s}}\right)$. When a constant external field, $H$, is applied, Young's modulus at the constant field, $E_{\mathrm{H}}$, of the ribbon on which $\sigma_{x}$ is applied is given as

$$
\frac{1}{E_{H}}=\frac{1}{E_{m}}+\frac{9 \lambda_{s}^{2} H^{2}}{M_{s} H_{\mathrm{a} \sigma}^{3}}=\frac{1}{E_{m}}+\frac{M_{s}{ }^{2} H^{2}}{3 \lambda_{s}\left(\sigma_{c}-\sigma_{x}\right)^{3}}
$$

Equation (2) is only valid for the former case because the magnetic easy axis will remain in the width direction as long as $\sigma_{x}<\sigma_{\mathrm{c}}=2 K_{\mathrm{u}} / 3 \lambda_{\mathrm{s}} \sigma_{x}$, but will abruptly switch to the length direction for $\sigma_{x}>\sigma_{c}$. In the latter case, $E_{\mathrm{H}}$ will be $E_{\mathrm{m}}$. Substituting $E_{\mathrm{H}}$ from Eq. (2) into Eq. (1) for the modulus of elasticity describes the resonance frequency, $f_{\mathrm{H}}$, as functions of the applied field of $H$ and/or applied stress, $\sigma_{x}$;

$$
\begin{aligned}
& f_{\mathrm{H}}=f_{\text {Mech }}\left(1+\frac{9 \lambda_{s}^{2} E_{m}{ }^{2} H^{2}}{M_{s} H_{\mathrm{a} \sigma}^{3}}\right)^{-\frac{1}{2}}=f_{\text {Mech }}\left(1+\frac{M_{s}{ }^{2} H^{2}}{3 \lambda_{s}\left(\sigma_{c}-\sigma_{x}\right)^{3}}\right)^{-\frac{1}{2}} \\
& f_{\text {Mech }}=\frac{1}{2 L} \sqrt{\frac{E_{m}}{\rho}}
\end{aligned}
$$

where $f_{\text {Mech }}$ denotes the purely mechanical, or elastic, contribution to $f_{\mathrm{H}}$. The magnetoelastic properties that lead to the $\Delta E$ effect have been separated from the mechanical properties, and Eq. (3) readily separates into two factors describing the elastic and magnetoelastic contributions to $f_{\mathrm{H}}$.

The theoretical variations of magnetization, $M$, and $f_{H}$ with $H$ derived from Eq. (3) are schematically outlined in Fig. 3(a) and (b). It should be noted that inhomogeneities in anisotropy and other parameters in real materials will lead to rounded, instead of sharp, transitions. The $M-H$ curve for $0<\sigma_{x}<\sigma_{c}$ in Fig. 3(a) indicates that $M$ saturates to a constant value of $M_{\mathrm{s}}$ as long as $H$ is higher than $H_{\mathrm{a}}-\left(3 \lambda_{\mathrm{s}} \sigma_{x} / M_{\mathrm{s}}\right)$. The corresponding $f_{\mathrm{H}}$ value for $0<\sigma_{x}<\sigma_{\mathrm{c}}$ also saturates to $f_{\text {Mech }}$ at $H>H_{\mathrm{a}}-\left(3 \lambda_{\mathrm{s}} \sigma_{x} / M_{\mathrm{s}}\right)$ as seen in Fig. 3(b). In contrast, the $M$ value for $\sigma_{x}<0$ is lower than that for $\sigma_{x}=0$ when $H$ is lower than $H_{\mathrm{a}}+\left(3 \lambda_{\mathrm{s}}\left|\sigma_{x}\right| / M_{\mathrm{s}}\right)$ as seen in Fig. 3(a). This leads to an increase in $f_{\mathrm{H}}$ at $H=H_{\mathrm{a}}$ as seen in Fig. 3(b). The $f_{\mathrm{H}}$ ap proaches $f_{\text {Mech }}$ at $H=H_{\mathrm{a}}$ in the results by applying tensile, $\sigma_{x}>0$, and/or compressive, $\sigma_{x}<0$, stresses.

There are tensile and compressive stresses in the outer and inner bending zones on both sides of the neutral plane in a curved ribbon and the stress distribution is symmetrical; the absolute values of the stresses increase linearly with the distance from the neutral plane and are proportional to the corresponding curvature. Magnetization in the outer bending zone saturates in the longitudinal direction of the ribbon, and $f_{\mathrm{H}}=f_{\text {Mech }}$ at $H=H_{\mathrm{a}}$. In contrast, $\sigma_{x}<0$ and $\lambda_{\mathrm{s}} \sigma_{x}<0$ in the inner bending zone, which enhances the anisotropy field to $H_{\mathrm{a}}+\left(3 \lambda_{\mathrm{s}}\left|\sigma_{x}\right| / M_{\mathrm{s}}\right)$ and results in an increase in $f_{\mathrm{H}}$ at $H=H_{\mathrm{a}}$ The $f r\left(=f_{\mathrm{H}}\right)$ at $H_{\mathrm{dc}}(=H)=H_{\mathrm{a}}$ in our study was greatly increased by bending the ribbon, which weakened the $\Delta E$
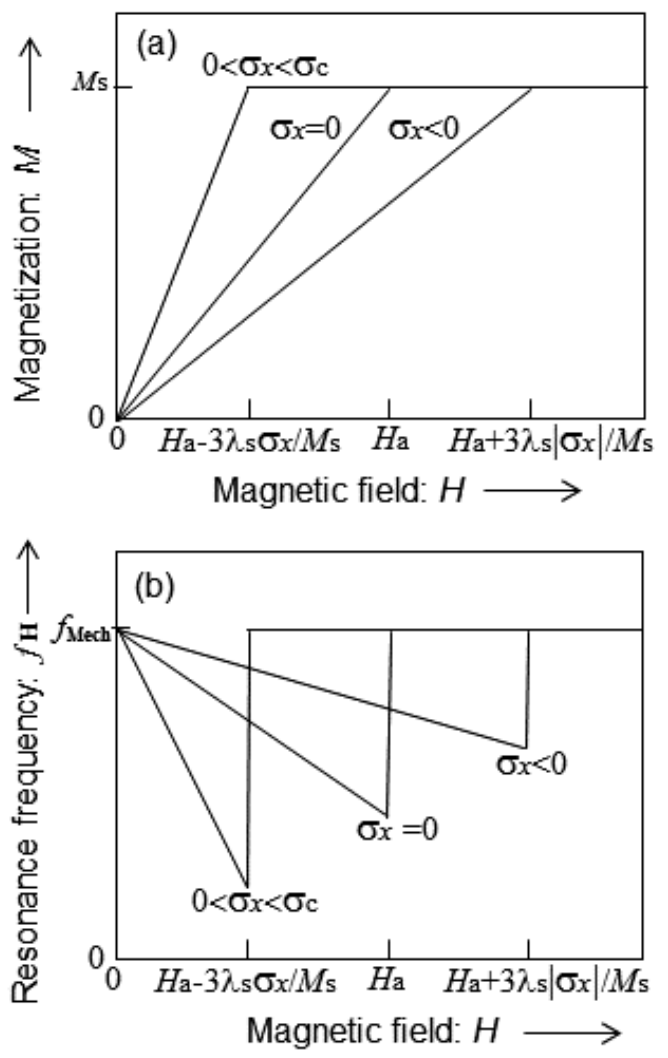

Fig. 3 Results of idealized model. (a) Variation of longitudinal magnetization, $M$, with longitudinal field, $H$, at various values of longitudinal stress, $\sigma x$. (b) Variation of resonance frequency, $f \mathrm{H}$, with $H$ at various values of $\sigma x$. 


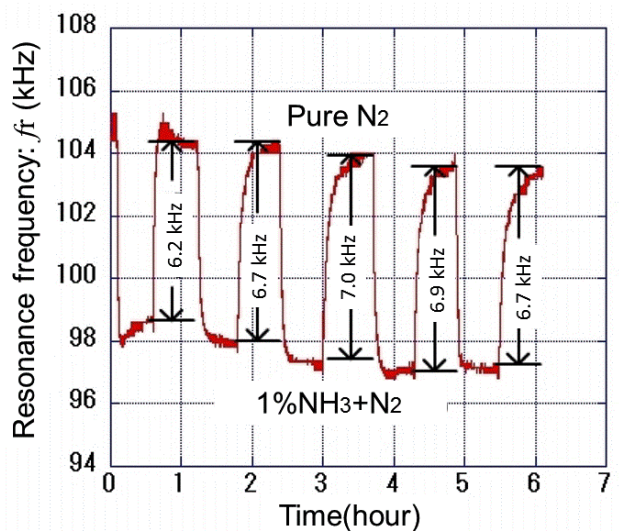

Fig. 4 Time response of the sensor to periodic cycles from a pure nitrogen atmosphere to a mixture of $1 \%$ ammonia in nitrogen.

effect.

Figure 4 plots the time response of $f r$ to periodic cycles where the atmosphere was changed from pure nitrogen $\left(P_{\mathrm{am}}=0 \%\right)$ to a mixture of $1 \%$ ammonia in nitrogen. The frequency shift, $\Delta f$, ranged from 6.2 to $7.2 \mathrm{kHz}$, and the average $\Delta f$ value was $6.7 \mathrm{kHz}$. As the number of cycles increased, the $f r$ measured in pure nitrogen gradually decreased from $105 \mathrm{kHz}$ in the first cycle to $\sim 103.5 \mathrm{kHz}$ in the fourth or later ones indicating that the sensitivity of this sensor should be stabilized by exposure to a high $P$ am atmosphere prior to practical use. The error in measurement of $P_{\mathrm{am}}$ was estimated to be about $0.1 \%$ from these results. Since commercially available ceramic ammonia sensors have much greater sensitivity (several ppm)1), further improvements to sensitivity are needed to put PEDOT/PSS-coated ribbons into practical use. For example, the thickness of PEDOT/PSS film should be increased to enhance the $\Delta f$ value as well as to improve sensitivity. The average $90 \%$ response time, $T_{90}$, in steps from 0 to $1.0 \%$ ammonia was $500 \mathrm{sec}$, so the $f r$ and $1 / R$ values were measured after holding the target, $P_{\mathrm{am}}$, for $10 \mathrm{~min}$.

Figure 5 plots the relation between ammonia concentration, $P_{\mathrm{am}}$, and $T_{90}$. The $T_{90}$ changes linearly from $1600 \mathrm{~s}$ at $P_{\mathrm{am}}=0.1 \%$ to $200 \mathrm{~s}$ at $P_{\mathrm{am}}=10 \%$, which is longer than the values for commercially available ammonia sensors $\left(T_{90}=\text { several to several tens of seconds }\right)^{1}$. However, it was considered to be sufficiently short for sensors to monitor the atmosphere in food packages, because the deterioration of food is generally the result of rather slow chemical reactions. It generally takes several hours or more before food decays and begins to emit an unpleasant smell under ordinary conditions.

Figure 6 plots the dependence of the $1 / R$ and $f r$ of the PEDOT/PSS-coated magnetic ribbon on $P_{\text {am }}$. When $P_{\text {am }}$ was zero (pure nitrogen atmosphere), the PEDOT/PSS film contracted and the ribbon noticeably bent, resulting in the largest $1 / R$. In contrast, as $P_{\text {am }}$ increased, the PEDOT/PSS film expanded and $1 / R$ decreased to $27 \mathrm{~m}^{-1}$ at $P_{\mathrm{am}}=10 \%$. The $\mathrm{fr}$ value of the ribbon decreased $\mathrm{mo}^{-}$ notonously from $105 \mathrm{kHz}$ at $P_{\mathrm{am}}=0 \%$ to $89.0 \mathrm{kHz}$ at $P_{\mathrm{am}}=$ $10 \%$.

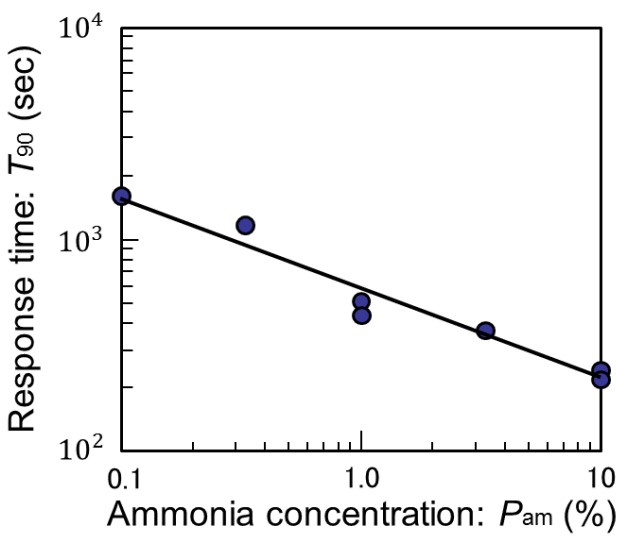

Fig. 5 Relation between ammonia concentration, $P_{\text {am }}$, and $90 \%$ response time, $T_{90}$.

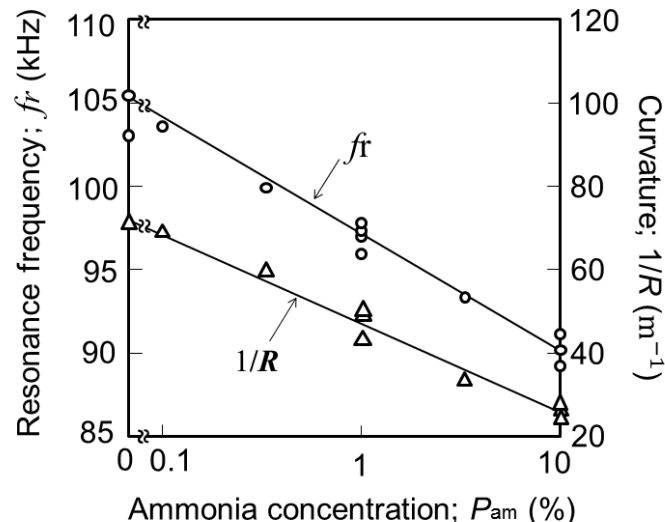

Fig. 6 Ammonia concentration, $P$ am, dependence of resonance frequency, $f r$, and curvature, $1 / R$.

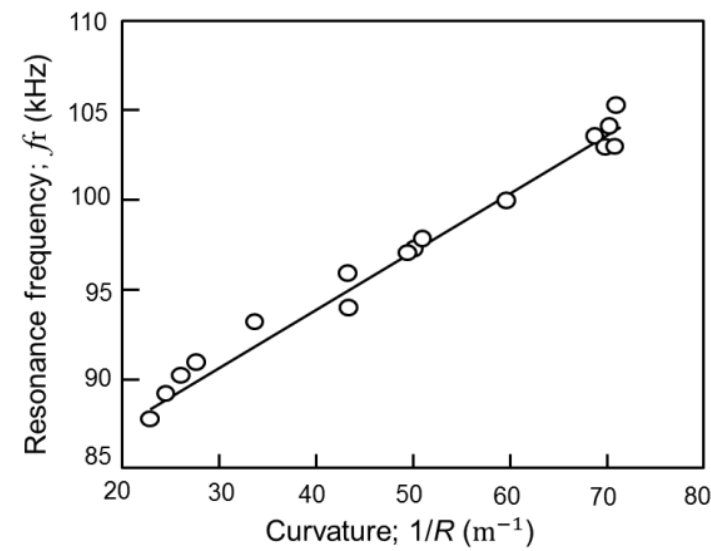

Fig. 7 Relation between curvature, $1 / R$, and resonance frequency, $f r$.

Figure 7 plots the relation between $f r$ and $1 / R$. We clarified that the $f r$ value was almost linear in relation to $1 / R$, and its proportional constant was about 0.33 $\mathrm{kHz} / \mathrm{m}^{-1}$. As previously mentioned, applications of tensile and compressive stresses that were proportional to $1 / R$, resulted in reducing the $\Delta E$ effect and increasing $f r$. A similar dependence of $f r$ on $1 / R$ has been reported for a PdNi-coated magnetic ribbon hydrogen sensor ${ }^{2}$, a TiNiCu-coated magnetic ribbon actuator ${ }^{11)}$, and a PEDOT/PSS-coated magnetic ribbon humidity sensor ${ }^{3)}$. 
Since PEDOT/PSS expands or contracts when it absorbs or desorbs water vapor ${ }^{5}$ ) or ammonia molecules, the PEDOT/PSS-coated magnetic ribbon responds to both gases. It is preferable to have an additional wireless humidity sensor to measure $P_{\mathrm{am}}$ in the presence of water vapor. An array of multiple magnetic ribbons, each having different lengths and non-overlapping operational frequency ranges and coated with a different gas sensing layer, can be used for this purpose to simultaneously measure multiple gas concentrations, such as humidity and $P_{\mathrm{am}}$. For example, a humidity sensor can be fabricated by coating a magnetic ribbon with a thin film that is highly responsive to humidity, such as $\mathrm{Al}_{2} \mathrm{O}_{3}{ }^{4}$. . Using the output of this sensor to calibrate for humidity, an absolute $P_{\mathrm{am}}$ value can be determined from the PEDOT/PSS-coated sensor.

The above results revealed that $\Delta f$ and $T_{90}$ had opposing tendencies with respect to $P_{\text {am }}$, which suggests that the sensitivity and response time of the sensors have a tradeoff relationship. To achieve a wireless ammonia sensor for practical use, we must optimize the $\Delta f$ and $T_{90}$ values. Further investigations are now under way to optimize specifications such as the PEDOT/PSS layer thickness and the ribbon dimensions toward simultaneously increasing $\Delta f$ and reducing $T_{90}$.

\section{Conclusion}

We investigated a wireless ammonia sensor that employs the change in the mechanical resonance frequency, $f r$, in a magnetic ribbon coated with poly(3,4-ethylenedioxythiophene) doped with poly(4-styrenesulfonate) (PEDOT/PSS). As PEDOT/PSS expands or contracts when it absorbs or desorbs ammonia molecules, the curvature, $1 / R$, of the ribbon varies in response to changes in the ammonia concentration, $P$ am. We also proved that $f r$ is proportional to $1 / R$. Consequently, $P_{\mathrm{am}}$ was able to be detected by monitoring fr using a pickup coil placed away from the ribbon; the $f r$ value changed from $89 \mathrm{kHz}$ at $P_{\mathrm{am}}=10 \%$ to $105 \mathrm{kHz}$ at $P_{\mathrm{am}}=0 \%$. The $90 \%$ response time changed linearly from $1600 \mathrm{~s}$ at $P_{\mathrm{am}}=$ $0.1 \%$ to $200 \mathrm{~s}$ at $P_{\mathrm{am}}=10 \%$. The magnetic ribbons are inexpensive, so the sensors are disposable. Consequently, we confirmed the enormous potential of the ribbons by employing them as wireless ammonia sensors, which can be used for monitoring the condition of food in airtight and tamper-resistant packages.

Acknowledgement Support of this work from the Japan Science and Technology Agency under grant (No. AS221Z0364B) is gratefully acknowledged.

\section{References}

1) http://www.figaro.co.jp/product/index.

2) T. Shibata, N. Kutsuzawa, S. Yoshizawa, S. Kambe, and O. Ishii: J. Magn. Soc. Jpn., 33, 50 (2009).

3) O. Ishii, R. Kakehata, N. Kutsuzawa, and H. Okuzaki: IEEJ Trans. 7(S1), S190 (2012).

4) M. K. Jain, S. Schmidt, K. G. Ong, C. Mungle, and C. A.
Grimes: Smart Mater. Struct. 9, 502 (2000).

5) H. Okuzaki and T. Kunugi: J. Polym. Sci., Polym. Phys. 34 1742 (1996)

6) Q. Pei and O. Inganas: Synth. Met., 55-57, 3730 (1993).

7) H. Okuzaki, H. Suzuki, and T. Ito: J. Phys. Chem. B, 113, 11378 (2009).

8) J. D. Livingston: Phys. Stat. Sol. (a) 70, 591 (1982).

9) M. L. Spano, K. B. Hateway, and H. T. Savage: J. Appl. Phys. 53, 2867 (1982).

10) G. Herzer: J. Magn. Magn. Mater. 254-255, 598 (2003).

11) Y. Miyahara, O. Ishii, S. Kambe, N. Kutsuzawa, and A. Ishida: J. Magn. Soc. Jpn., 34, 584 (2010) (in Japanese).

Received Oct. 18, 2012; Revised Feb. 10, 2013; Accepted Mar. 7, 2013 